\title{
Calcium and Potassium Are Important Regulators of Barrier Homeostasis in Murine Epidermis
}

Seung Hun Lee, Peter M. Elias, Ehrhardt Proksch, Gopinathan K. Menon, Man Mao-Quiang, and Kenneth R. Feingold

Department of Medicine and Dermatology, University of California, San Francisco, San Francisco, California 94121; and the

Metabolism Section and Dermatology Department, Veterans Administration Medical Center, San Francisco, California, 94121

\begin{abstract}
Topical solvent treatment removes lipids from the stratum corneum leading to a marked increase in transepidermal water loss (TEWL). This disturbance stimulates a variety of metabolic changes in the epidermis leading to rapid repair of the barrier defect. Using an immersion system we explored the nature of the signal leading to barrier repair in intact mice. Initial experiments using hypotonic to hypertonic solutions showed that water transit per se was not the crucial signal. However, addition of calcium at concentrations as low as $0.01 \mathrm{mM}$ inhibited barrier repair. Moreover, both verapamil and nifedipine, which block calcium transport into cells, prevented the calcium-induced inhibition of TEWL recovery. Additionally, trifluroperazine or $\mathbf{N}$-6-aminohexyl-5-chloro-1-naphthalenesulfonamide, which inhibit calmodulin, prevented the calcium-induced inhibition of TEWL recovery. Although these results suggest an important role for calcium in barrier homeostasis, calcium alone was only modestly effective in inhibiting TEWL recovery. Potassium alone $(10 \mathrm{mM})$ and phosphate alone $(5 \mathrm{mM})$ also produced a modest inhibition of barrier repair. Together, however, calcium and potassium produced a synergistic inhibition of barrier repair (control $50 \%$ recovery vs. calcium + potassium 0-11\% recovery in $2.5 \mathrm{~h}$ ). Furthermore, in addition to inhibiting TEWL recovery, calcium and potassium also prevented the characteristic increase in 3-hydroxy-3-glutaryl CoA reductase activity that occurs after barrier disruption. Finally, the return of lipids to the stratum corneum was also blocked by calcium and potassium. These results demonstrate that the repair of the epidermal permeability barrier after solvent disruption can be prevented by calcium, potassium, and phosphate. The repair process may be signalled by a decrease in the concentrations of these ions in the upper epidermis resulting from increased water flux leading to passive loss of these ions. ( J. Clin. Invest. 1992. 89:530-538.) Key words: epidermis • stratum corneum • calcium channels • lipid synthesis • transepidermal water loss
\end{abstract}

\section{Introduction}

The major function of the epidermis is to form a protective layer, the stratum corneum, which prevents the excessive loss of bodily fluids, a requirement for terrestrial life. Although lipids account for only a small percentage of total stratum cor-

Address correspondence to Kenneth R. Feingold, M.D., Metabolism Section (111F), Veterans Administration Medical Center, 4150 Clement Street, San Francisco, CA 94121.

Received for publication 30 August 1990 and in revised form 4 Oc tober 1991

The Journal of Clinical Investigation, Inc

Volume 89, February 1992, 530-538 neum weight, they are crucial for the provision of the permeability barrier $(1,2)$. Topical solvent treatment results in the removal of stratum corneum lipids, which leads to a marked disruption in barrier function (3-5). Homeostatic repair mechanisms lead very rapidly to the return of stratum corneum lipids and the recovery of barrier function (4). An increase in epidermal cholesterol and fatty acid synthesis occurs in association with this recovery of barrier structure and function (3-5). The stimulation in cholesterol synthesis is accounted for by an increase in both the total activity and activation state of 3-hydroxy-3-glutaryl (HMG) CoA reductase ${ }^{1}(6)$, the rate-limiting enzyme of cholesterol synthesis (7). Moreover, inhibition of cutaneous cholesterol synthesis with topical lovastatin, a competitive inhibitor of HMG CoA reductase, impairs barrier recovery due to impaired formation and secretion of epidermal lamellar bodies, as well as delayed return of cholesterol to the stratum corneum (8).

While these results indicate that barrier requirements regulate epidermal lipid synthesis, recent studies suggest that the signal for the repair of the barrier following solvent disruption involves transepidermal water loss (4). If after disrupting the barrier, animals are covered with a water vapor-impermeable membrane that artificially restores barrier function, the usual increase in epidermal lipid synthesis is prevented and the return of lipids to the intercellular spaces of the stratum corneum is inhibited. Moreover, the abnormality in barrier function remains equal to or greater than that observed immediately after solvent treatment. In contrast, if after disrupting the barrier, animals are covered with a water vapor-permeable membrane, the usual increase in epidermal lipid synthesis is observed, stratum corneum lipid content returns towards normal, and, most importantly, barrier function recovers normally (4). These results suggests that water flux plays a role in mediating the repair response.

The aim of this study was to explore in further detail the nature of the signal that leads to the rapid repair of barrier structure and function following solvent induced disruption of the barrier. To address the issue of extracellular regulation, we developed an experimental model that combines many of the characteristics of an in vitro system with the advantages of studies in the intact animal. After barrier perturbation, the animal is suspended so that the treated side is immersed in experimental solutions. This results in the incubation of the viable epidermis of the intact animal in specific solutions, allowing a degree of uniformity and control that is comparable to that achieved in in vitro systems.

1. Abbreviations used in this paper: HMG CoA reductase, 3-hydroxy-3glutaryl CoA reductase; TEWL, transepidermal water loss; TMB-8, 3,4,5-trimethoxybenzoic acid 8-[diethy amino] octyl ester; W7, $\mathrm{N}$-6aminohexyl-5-chloro-1-napthalenesulfon-amide. 


\section{Methods}

Materials. Hairless male mice $(\mathrm{hr} / \mathrm{hr}) \mathrm{8}-10$ wk old were purchased from Jackson Laboratory, Bar Harbor, ME. They were fed mouse diet (Simonsen Laboratories, Inc., Gilroy, CA) and water ad lib. The ages ranged between 10 and 12 wk at the time of study. Acetone was purchased from Fisher Scientific Co., Pittsburgh, PA. Nile red was purchased from PolySciences, Inc., Warrington, PA. ${ }^{14} \mathrm{C}-\mathrm{HMG}$ CoA $(54.2$ $\mathrm{mCi} / \mathrm{mM})$ and tritiated mevalonic acid were purchased from New England Nuclear, Boston, MA. Anion exchange silica gels (AG1-X8, formate form, 200-400 mesh) were purchased from Bio-Rad Laboratories, Richmond, CA. EGTA, TMB-8 (3,4,5-trimethoxybenzoic acid 8-[diethy amino]octyl ester), trifluoperazine, verapamil, W7 ( $N$-6aminohexyl-5-chloro-1 naphthalenesulfonamide), and nifedipine were obtained from Sigma Chemical Co., St. Louis, MO. PBS contained 138 $\mathrm{mM} \mathrm{NaCl}, 8.1 \mathrm{mM} \mathrm{Na} \mathrm{HPO}_{4}, 2.7 \mathrm{mM} \mathrm{KCl}$, and $1.1 \mathrm{mM} \mathrm{KH} \mathrm{PO}_{4}$ (pH 7.4).

Experimental procedures. To acutely perturb barrier function the flanks of hairless mice, which were anesthetized with i.p. chloral hydrate and/or ether inhalation anesthesia, were gently treated with acetone-soaked cotton balls as described in previous publications (3-5). Acetone treatment does not result in visible or microscopic damage to the stratum corneum. Immediately after acetone treatment the skin was warmed to $\sim 35^{\circ} \mathrm{C}$ and transepidermal water loss (TEWL) was measured using an electrolytic water analyzer (Meeco Inc., Warrington, PA) as described previously (3-5). Animals with TEWL rates between 5 and $9 \mathrm{mg} / \mathrm{cm}^{2}$ per $\mathrm{h}$ were included in the study.

After disruption of the barrier with acetone, animals were immediately immersed with one flank submerged in various solutions for 2.5 $h$. The animals were suspended on wire netting to avoid contact with the petri dish container. The solution temperature was initially at $4^{\circ} \mathrm{C}$ for the first $15 \mathrm{~min}$ to allow for equilibration. Studies have demonstrated that cold temperature inhibits barrier recovery (Feingold, K. R., and P. M. Elias, unpublished observations). The temperature of these solutions was then constantly maintained at $\sim 35^{\circ} \mathrm{C}$. In addition, some animals were left exposed to air while other animals were covered with a tightly fitted water-impermeable membrane (one finger of a latex glove) immediately after topical acetone treatment. Before remeasuring transepidermal water loss, the skin surface was dried and warmed for $5 \mathrm{~min}$ with a heating lamp. In some experiments the animals were killed and skin samples obtained.

Histochemical staining. Nile red, a fluorescent probe for lipids, was used to demonstrate the distribution and content of lipids in the stratum corneum as described previously (4).

$H M G$ CoA reductase activity. The epidermis was separated from the dermis by immersion in $10 \mathrm{mM}$ EDTA and Dulbecco's PBS at $37^{\circ} \mathrm{C}$ for $40 \mathrm{~min}$ as described previously (6). The epidermis was homogenized in $0.3 \mathrm{M}$ sucrose, $10 \mathrm{mM}$ mercaptoethanol, $10 \mathrm{mM}$ EDTA, and
$50 \mathrm{mM}$ sodium chloride ( $\mathrm{pH} 7.4$ ), microsomes were isolated, and HMG CoA reductase activity in the microsomes determined, as described previously (6). HMG CoA reductase activity was expressed as nanomoles of mevalonate synthesized per milligram protein per minute. Protein was determined using the Bio-Rad Laboratories protein assay dye reagent.

Statistical significance was determined using a two-tailed Student's $t$ test.

\section{Results}

Role of water transit in barrier recovery. As illustrated in Fig. 1, if animals are left exposed to air after acetone disruption of the barrier, barrier function as measured by transepidermal water loss recovers by $\sim 50 \%$ in $2.5 \mathrm{~h}$. However, if the animals are covered with a water vapor impermeable latex membrane, which artificially restores barrier function, recovery does not occur. These results are similar to those previously reported by this laboratory (3-5).

Since our prior studies suggested that water transit is the signal for barrier recovery, our initial experiments were designed to determine whether an increase in water transit modulates the rate of barrier recovery after acute barrier disruption. Acetone-treated flanks were immersed in either isoosmolar sucrose or isotonic sodium chloride, solutions that should result in little or no net transit of water through the disrupted barrier. As shown in Fig. 1, the recovery of barrier function under these conditions is similar to that observed in air exposed animals.

We next assessed whether varying the osmolarity from hypotonic to hypertonic would influence the rate of barrier recovery. After the barrier is disrupted, immersion in solutions with a low osmolarity should lead to net transit of water into the epidermis, while solutions with a high osmolarity should induce a net transit of water out of the epidermis. As shown in Fig. 2, upper panel, there is no effect of varying the concentration of sodium chloride from zero (distilled water) to mildly hypertonic (190 mM) on barrier recovery. More hypertonic saline solutions ( $>200 \mathrm{mM}$ ) resulted in toxicity, and therefore could not be tested. Likewise, as shown in Fig. 2, lower panel, varying the osmolarity with sucrose over a wide range also did not affect barrier recovery. These results suggest that water transit across the stratum corneum alone is not the crucial signal that regulates barrier recovery.

Role of ions in barrier recovery. Since modulations of water transit did not influence barrier recovery, we next explored the effect of various ions on barrier recovery. As shown in Fig. 3, if

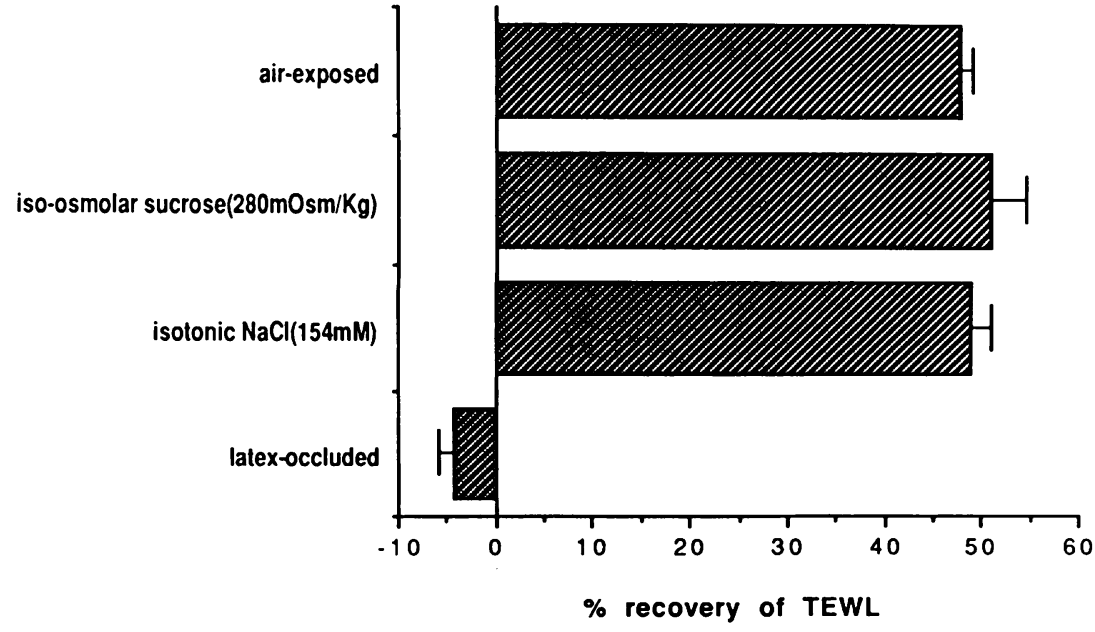

Figure 1. Barrier recovery after acetone disruption. After acetone disruption of the barrier, hairless mice were left exposed to air, covered with a water-impermeable latex membrane, or immersed in an isoosmolar sucrose or isotonic $\mathrm{NaCl}$ solution for $2.5 \mathrm{~h}$. TEWL was determined after the skin surface was dried and warmed. The results are expressed as the percentage recovery of barrier function (TEWL at $2.5 \mathrm{~h} / \mathrm{TEWL}$ immediately after acetone treatment) and presented as the mean \pm SEM. $N=4-7$ in each group. 

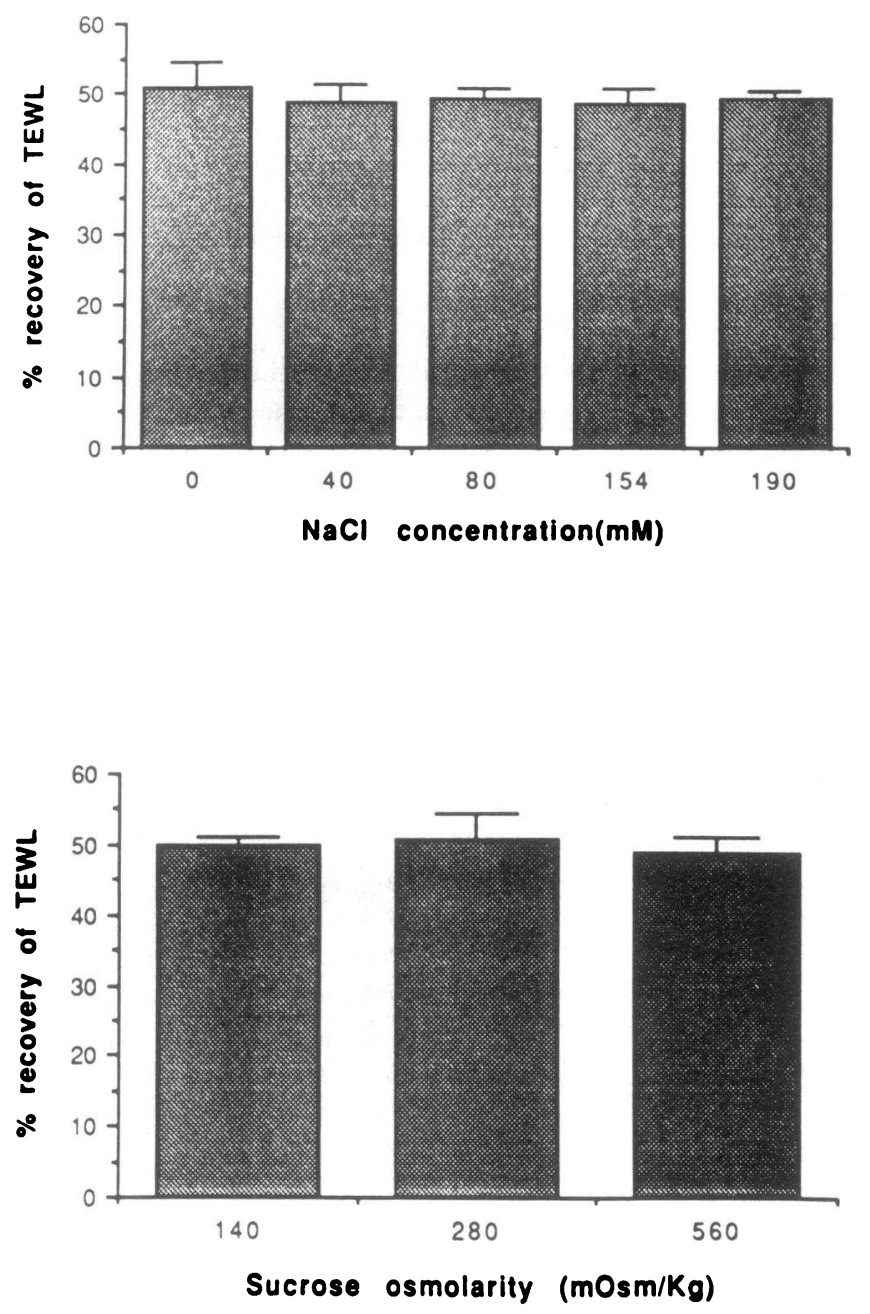

Figure 2. (A) The effect of sodium chloride concentration on barrier recovery. After acetone disruption of the barrier, hairless mice were immersed in varying concentrations of $\mathrm{NaCl}(0-190 \mathrm{mM})$ for $2.5 \mathrm{~h}$ followed by determination of TEWL. The results are expressed and presented as in Fig. 1. $N=4-7$ in each group. $(B)$ The effect of osmolarity on barrier recovery. After acetone disruption of the barrier, hairless mice were immersed in varying concentrations of sucrose $(140 \mathrm{mOSm} / \mathrm{kg}-560 \mathrm{mOSm} / \mathrm{kg}$ ) for $2.5 \mathrm{~h}$ followed by determination of TEWL. Data are presented and expressed as in Fig. 1. $N=4-7$ for each group. one adds calcium chloride alone to either isoosmolar sucrose or isotonic sodium chloride there is a modest decrease in barrier recovery. The maximum calcium effect is seen at $0.1 \mathrm{mM}$, and the half-maximal effect is seen with $\sim 0.01 \mathrm{mM}$. Likewise, if one adds $40 \mathrm{mM} \mathrm{KCl}$ alone to isoosmolar sucrose, a decrease in the recovery of barrier function occurs, which is similar in magnitude to that observed with calcium alone (Fig. 3). Most importantly, a combination of potassium $(40 \mathrm{mM})$ and calcium results in a synergistic inhibition of barrier recovery, which was maximal at $0.1 \mathrm{mM}$ calcium (Fig. 3).

Since the concentration of potassium $(40 \mathrm{mM})$ used in these experiments is supraphysiologic, we next studied barrier recovery in calcium- and magnesium-free PBS, a solution representative of extracellular fluid. PBS, which contains potassium but no calcium, by itself diminished barrier recovery to an extent similar to that observed with potassium alone (Fig. 3). Moreover, the addition of calcium to calcium- and magnesium-free PBS greatly inhibited barrier recovery, with a maximal effect observed at $0.1 \mathrm{mM}$ and a half-maximal effect at $0.01 \mathrm{mM}$. These results suggest that certain cations, in particular calcium, potassium, and phosphate, perhaps play a role in modulating barrier recovery.

To further characterize the specificity of the calcium effect we next assessed the effect of another divalent cation, magnesium, on barrier recovery. Whereas the addition of magnesium chloride to isoosmolar sucrose produced a slight decrease in barrier recovery, which did not achieve statistical significance, the addition of magnesium to calcium- and magnesium-free PBS resulted in a significant decrease in barrier recovery (Fig. 4). However, the effect of magnesium was much less than that observed with similar concentrations of calcium.

Fig. 5 shows the effect of various concentrations of potassium \pm calcium on barrier recovery. As described above, the addition of potassium alone to isoosmolar sucrose had a modest effect on barrier recovery, while the addition of potassium to isoosmolar sucrose containing $1.0 \mathrm{mM}$ calcium caused a dramatic inhibition of barrier repair (Fig. 5). In both circumstances, maximal inhibition of barrier recovery occurred at a concentration of $10 \mathrm{mM}$ potassium, and higher concentrations did not cause a greater effect.

The effect of phosphate alone on barrier recovery is shown in Fig. 6. Increasing concentrations of phosphate result in an inhibition in barrier recovery with maximum effects observed

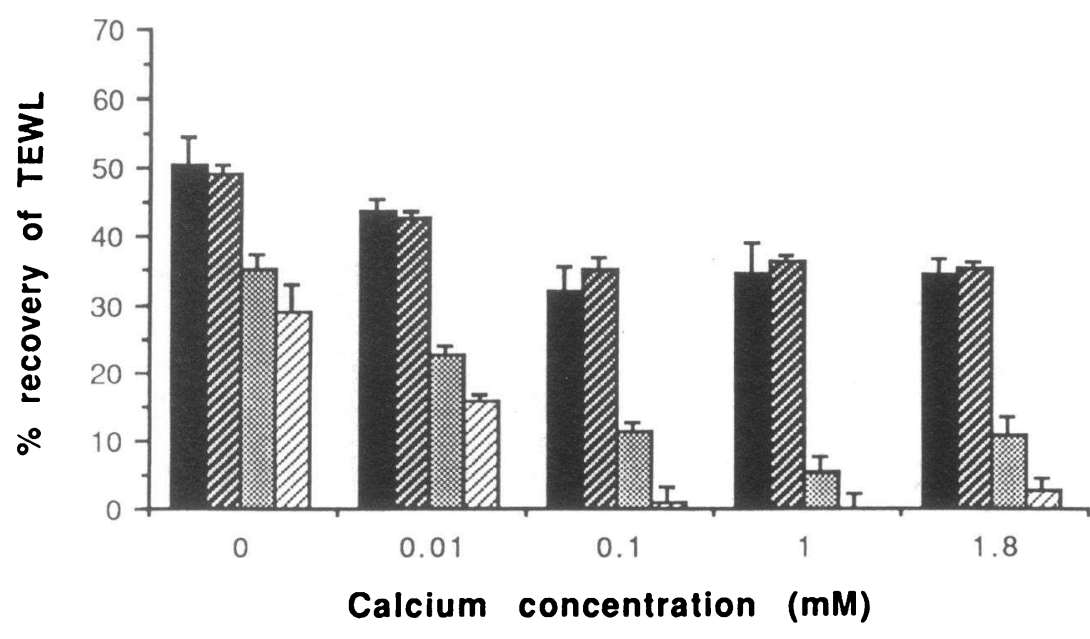

Figure 3. The effect of calcium on barrier recovery. After acetone disruption of the barrier, mice were immersed in isoosmolar sucrose, $190 \mathrm{mM}$ $\mathrm{NaCl}$, isoosmolar sucrose containing $40 \mathrm{mM}$ $\mathrm{KCL}$, or calcium-, magnesium-free PBS. The concentration of $\mathrm{CaCl}_{2}$ was varied from $0-1.8 \mathrm{mM}$ and after $2.5 \mathrm{~h}$ TEWL was determined. Data are presented and expressed as in earlier figures. $N$ = 4-7 for each group. $₫$, isoosmolar sucrose; $\square$, $\mathrm{NaCl}(190 \mathrm{mM})$; $\mathrm{KCl}(40 \mathrm{mM})$;, PBS (Ca, $\mathrm{Mg}$ free). 


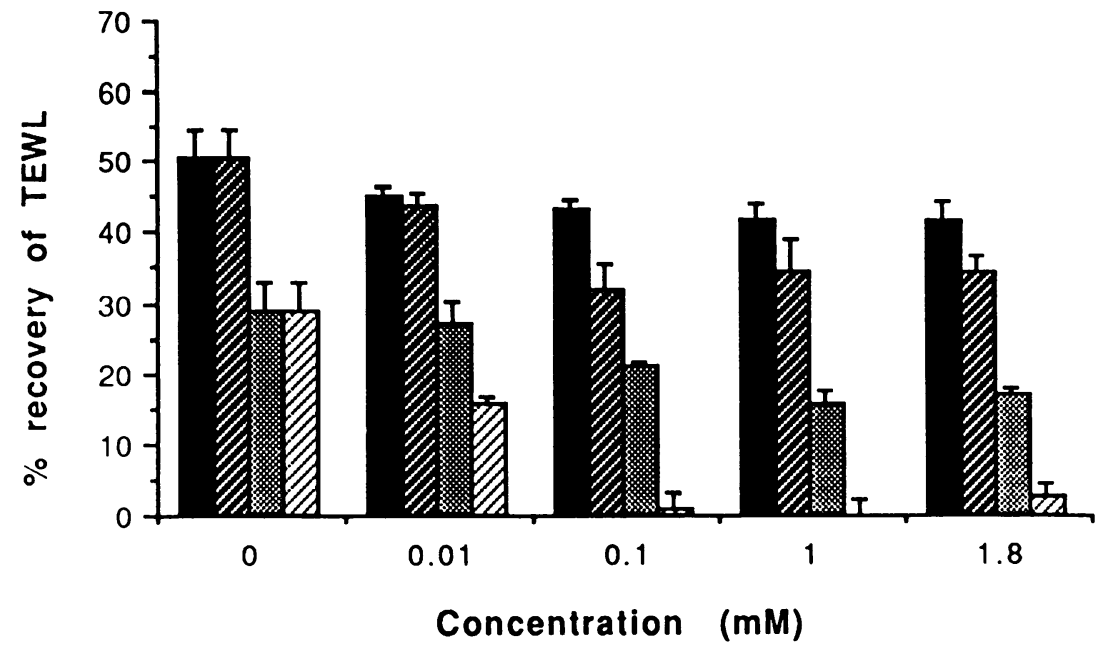

Figure 4. The effect of calcium or magnesium on barrier recovery. After acetone disruption of the barrier, mice were immersed in isoosmolar sucrose or calcium-, magnesium-free PBS containing varying concentrations of $\mathrm{MgCl}_{2}$ or $\mathrm{CaCl}_{2}$. After $2.5 \mathrm{~h}$ TEWL was determined. Data are presented and expressed as in earlier figures. $N=4-7$ for each group. $\mathrm{m}, \mathrm{Mg}^{++}$in isoosmolar sucrose; $\mathbf{m}, \mathrm{Ca}^{++}$ in isoosmolar sucrose; t, $^{\mathrm{Mg}^{++}}$in $\mathrm{PBS}$; 四, $\mathrm{Ca}^{++}$ in PBS. at $5 \mathrm{mM}$. The combination of phosphate $(9.5 \mathrm{mM})$ and potassium $(40 \mathrm{mM})$ did not result in a synergistic or additive inhibition of barrier recovery (recovery remained between 30 and $35 \%)$. The combination of phosphate $(9.5 \mathrm{mM})$ and calcium $(1.0 \mathrm{mM})$ resulted in a modest increase in the inhibition of barrier recovery $(23 \pm 2.9 \%, n=5)$ but not to the levels seen with PBS + calcium.

To demonstrate that the inhibition of barrier recovery by calcium- and potassium-containing solutions is not secondary to toxic effects, animals were exposed to air after immersion in PBS plus calcium for $2.5 \mathrm{~h}$ as in the studies described above. As shown in Fig. 7, the recovery of barrier function in animals exposed to air immediately after immersion (solid line and squares) is similar to animals exposed to air immediately after barrier disruption (dotted line and open squares). These results indicate that the inhibition of barrier recovery by calcium and/ or potassium cannot be attributed to nonspecific toxic effects.

Because of the apparent importance of calcium in mediating barrier repair after acetone disruption, we next compared barrier recovery in animals immersed in isoosmolar sucrose with and without $20 \mathrm{mM}$ EGTA, a compound that binds calcium with a high affinity. The recovery of barrier function is similar in both solutions (isoosmolar sucrose:50.8 $\pm 3.7 \%$ vs. isoosmolar sucrose $+20 \mathrm{mM}$ EGTA $49.0 \pm 2.3 \%$ recovery of transepidermal water loss at $2.5 \mathrm{~h}$; NS), indicating that binding of calcium does not accelerate the rate of recovery.

Effect of calcium channel and calmodulin inhibitors on barrier recovery. To determine the role of calcium influx into cells on barrier recovery we next employed inhibitors of calcium uptake. As shown in Fig. 8, verapamil, a calcium channel inhibitor (9), reversed the inhibition of barrier recovery that is caused by incubation in solutions containing calcium with or without potassium. Verapamil is effective at concentrations as low as $0.05 \mu \mathrm{M}$ with a maximal effect at $1 \mu \mathrm{M}$. Moreover, the modest effect of calcium alone on barrier recovery also is prevented by verapamil. Furthermore, nifedipine, which belongs to another class of calcium channel inhibitors (9), also prevents the inhibition of barrier recovery that is characteristically caused by calcium and potassium (Fig. 8). These results indicate that the cellular uptake of calcium plays a crucial role in regulating barrier homeostasis.

To determine the importance of cytosolic calcium we next determined the effect of TMB-8, a compound that inhibits the translocation of calcium from membrane storage sites to the cytosol (10). As shown in Fig. 9, TMB-8 allows barrier recovery to occur in the presence of calcium and potassium. These re-

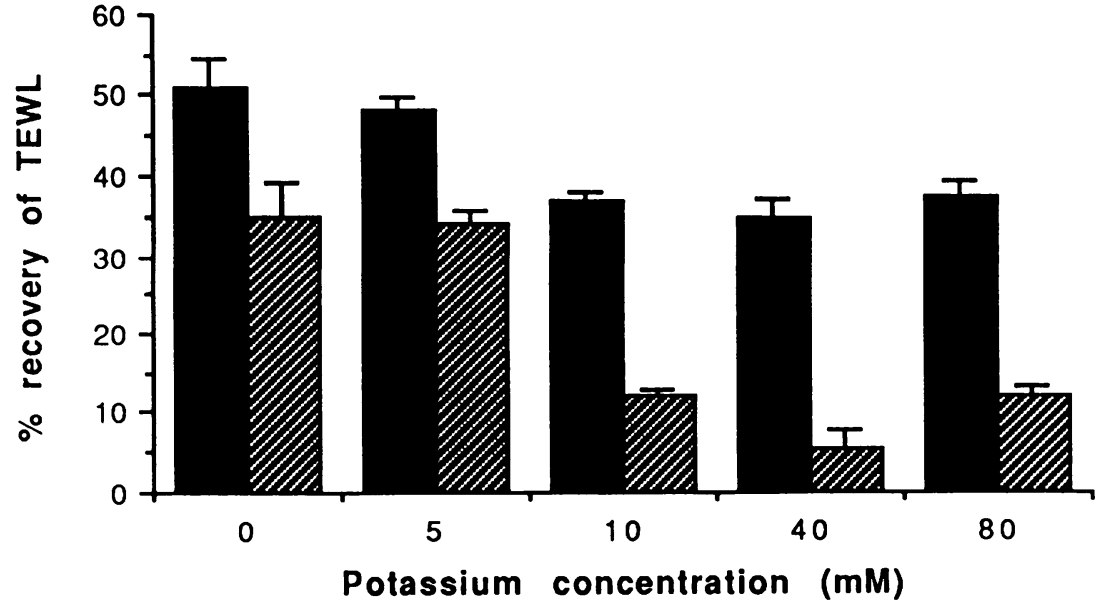

Figure 5. Effect of potassium on barrier recovery. After acetone disruption of the barrier, mice were immersed in isoosmolar sucrose or isoosmolar sucrose containing $1.0 \mathrm{mM} \mathrm{CaCl}_{2}$. The $\mathrm{KCL}$ concentration was varied from 0 to $80 \mathrm{mM}$ and after $2.5 \mathrm{~h}$ TEWL was determined. Data are presented and expressed as in earlier figures. $N=4-7$ for each group. $\mathrm{m}$, isoosmolar sucrose; $\mathrm{m}, 1.0 \mathrm{mM} \mathrm{Ca}^{++}$ in isoosmolar sucrose. 


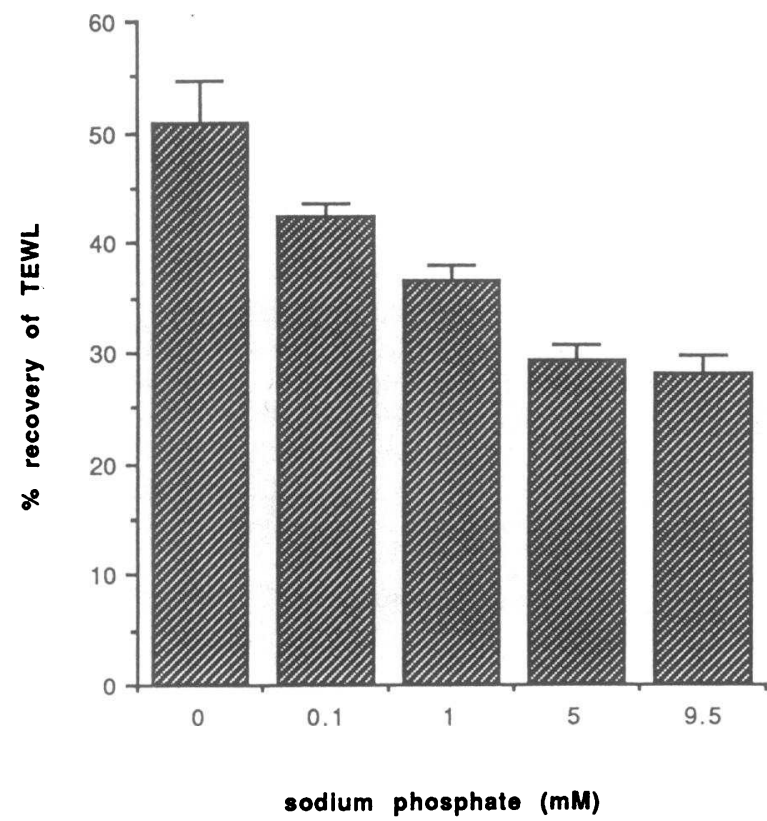

Figure 6. Effect of phosphate on barrier recovery. After acetone disruption of the barrier, mice were immersed in isoosmolar sucrose or isoosmolar sucrose containing various concentrations of sodium phosphate. After $2.5 \mathrm{~h}$ TEWL was determined. Data are presented and expressed as in earlier figures. $N=4-7$ for each group.

sults suggest that the localization of intracellular calcium plays a role in the inhibitory effects of calcium and potassium.

To assess one possible mechanism of the intracellular calcium induced inhibition, we next determined the effect of the calmodulin inhibitors, trifluroperazine and W7 $(11,12)$, on barrier recovery. The addition of either trifluroperazine or $\mathrm{W} 7$ prevents the inhibition of barrier recovery characteristically caused by calcium and potassium (Fig. 10). Both trifluroperazine and W7 prevent the inhibition at concentrations as low as $0.001 \mu \mathrm{M}$ with maximal effects at $1 \mu \mathrm{M}$. These results suggest that calmodulin plays a role in the calcium effect on barrier recovery.
HMG CoA reductase activity. Previous studies have shown that barrier recovery is dependent upon rapid increases in the de novo synthesis of cholesterol $(3-5,8)$. Therefore, to determine whether the effects of calcium and/or potassium on barrier recovery are linked to changes in lipid synthesis, we next determined the activity of HMG CoA reductase, the ratelimiting enzyme of cholesterol synthesis (7), after disruption of the barrier. As seen in Table I, the activity of HMG CoA reductase increases $65 \%$ in animals exposed to air. Whereas previous studies have demonstrated that this increase in HMG CoA reductase activity is prevented by latex occlusion (6), immersion in an isotonic sodium chloride solution does not influence the characteristic increase in HMG CoA reductase activity that follows barrier disruption (Table I). Immersion in calcium- and magnesium-free PBS solution results in a very slight decrease in enzyme activity, but this does not achieve statistical significance. In contrast, immersion in a PBS solution containing calcium completely inhibits the expected increase in HMG CoA reductase activity that occurs after barrier disruption. These results show that the differences in barrier recovery that occur in the various solutions is associated with parallel modulations in cholesterol synthesis.

Morphological studies. In previous studies we have demonstrated that acetone treatment removes stainable neutral lipids from the stratum corneum (4). Over time these neutral lipids return to the stratum corneum in parallel with barrier recovery (4). As shown in Fig. $11 \mathrm{~A}$, in the air-exposed animals, bright yellow fluorescence reappears, indicative of neutral lipid repletion of the stratum corneum. In animals treated with acetone and then occluded with a latex wrap, no return of stainable lipids occurs (Fig. $11 B$ ), results similar to those previously reported (4). In animals immersed in calcium- and magnesium-free PBS, partial return of lipid staining is apparent at 2.5 $\mathrm{h}$ (Fig. $11 \mathrm{C}$ ). In contrast, in animals immersed in PBS supplemented with $1.0 \mathrm{mM}$ calcium, the return of lipids to the stratum corneum is inhibited (Fig. $11 \mathrm{D}$ ).

\section{Discussion}

Previous studies have shown that topical solvent treatment results in the removal of lipids from the stratum corneum which

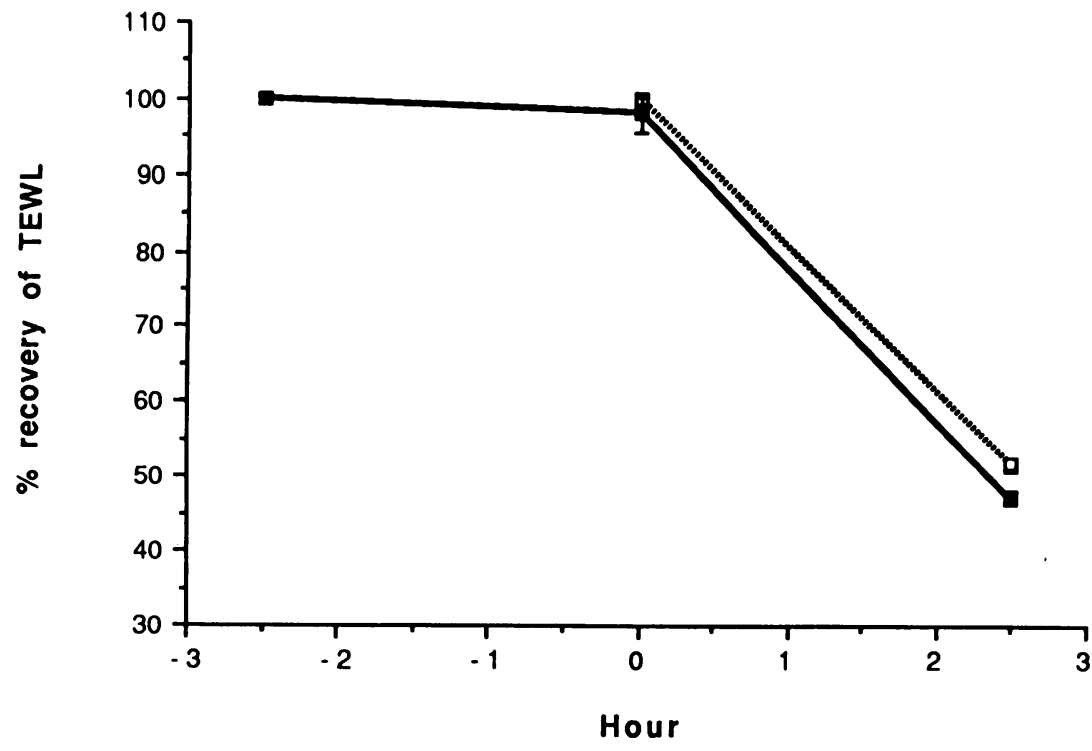

Figure 7. Barrier recovery following immersion. After acetone disruption of the barrier, mice were immersed in calcium-, magnesium-free PBS containing $1.8 \mathrm{mM} \mathrm{CaCl}_{2}$ for $2.5 \mathrm{~h}$. After immersion the animals were then exposed to air for $2.5 \mathrm{~h}$. Another set of animals was exposed to air immediately after barrier disruption. Data are presented and expressed as in earlier figures. $N=5$ for each group. ----- - -----, air exposed; $\longrightarrow-$, immersion-air exposed. 


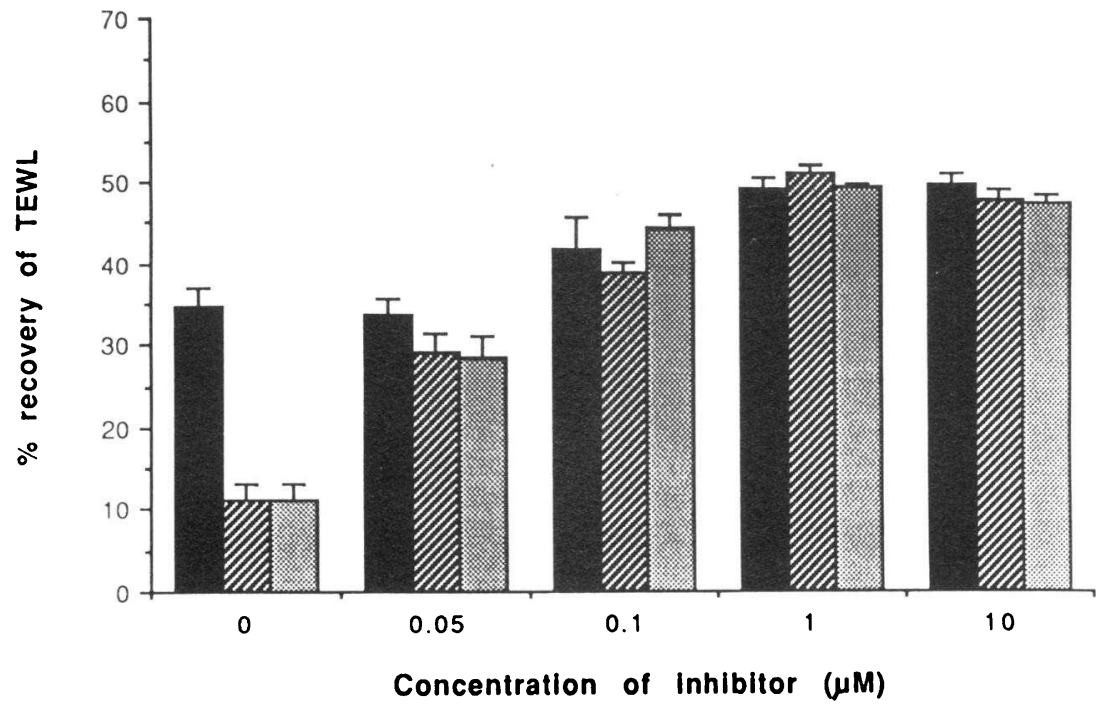

Figure 8. Effect of calcium channel blockers on barrier recovery. After acetone disruption of the barrier, mice were immersed in isoosmolar sucrose containing $1.8 \mathrm{mM} \mathrm{CaCl}_{2}$ alone or $1.8 \mathrm{mM} \mathrm{CaCl}_{2}$ $+40 \mathrm{mM} \mathrm{KCL}$. Various concentrations of verapamil or nifedipine were added as indicated and TEWL was determined at $2.5 \mathrm{~h}$. Data are presented and expressed as in earlier figures. $N=3-6$ for each group. $\mathrm{m}$, verapamil $+1.8 \mathrm{mM} \mathrm{Ca}^{++}$; verapamil $+40 \mathrm{mM} \mathrm{K}^{+}+1.8 \mathrm{mM} \mathrm{Ca}^{++}$; $\square$, nifedipine $+40 \mathrm{mM} \mathrm{K}^{+}+1.8 \mathrm{mM} \mathrm{Ca}^{++}$. leads to a marked disruption in barrier function (4). This perturbation stimulates a variety of metabolic changes in the epidermis, including accelerated formation and secretion of epidermal lamellar bodies, as well as increased cholesterol and fatty acid synthesis $(2-6,8)$. These changes in turn lead to the restoration of the lipid content of the stratum corneum and normalization of barrier function. The signal that initiates this repair process is unknown, but previous studies have suggested that transepidermal water loss might be an important factor (4). For example, if after disruption of the barrier, animals are covered with a water impermeable membrane, this prevents the expected increase in transepidermal water loss, the return of stratum corneum lipids, and barrier recovery does not occur (4). In contrast, if comparably treated animals are covered with a vapor-permeable membrane, which allows for an elevated transepidermal water loss, the stratum corneum lipid content and barrier function recover normally (4).

We initially determined whether preventing or altering water loss across the stratum corneum would influence barrier recovery. Neither an isoosmolar sucrose solution nor an isotonic sodium chloride solution, both of which decrease net water loss, alter barrier repair. Similarly, varying the osmolarity of the immersion solution from hypotonic (distilled water) to hypertonic ( $540 \mathrm{mOSm} / \mathrm{kg}$ sucrose), both of which should effect the rate and/or direction of net water flux, also did not alter barrier repair. These observations indicate that water transit per se is unlikely to be the crucial signal that stimulates barrier recovery following acute disruption of the barrier with solvents.

In contrast, our studies have demonstrated that specific ions (calcium, potassium, and phosphate) may be crucial in regulating barrier homeostasis. Addition of calcium at concentrations as low as $0.01 \mathrm{mM}-0.1 \mathrm{mM}$ to the immersion solution inhibited barrier repair. In contrast, magnesium, another divalent anion, also inhibited barrier recovery, but not as effectively as calcium. This effect is analogous to that of divalent cations on the parathyroid gland where both calcium and magnesium inhibit parathyroid hormone secretion but calcium is much more potent (13). Moreover, these studies are consistant with recent studies that demonstrate that calcium plays an important role in mediating keratinocyte function (14-18). In tissue culture the addition of calcium to the medium (concentrations as low as $0.1 \mathrm{mM}$ in mouse keratinocytes) results in inhibition of proliferation and terminal differentiation of both murine and human keratinocytes (14-19). Moreover, previous studies have demonstrated a calcium gradient in the epidermis in vivo,

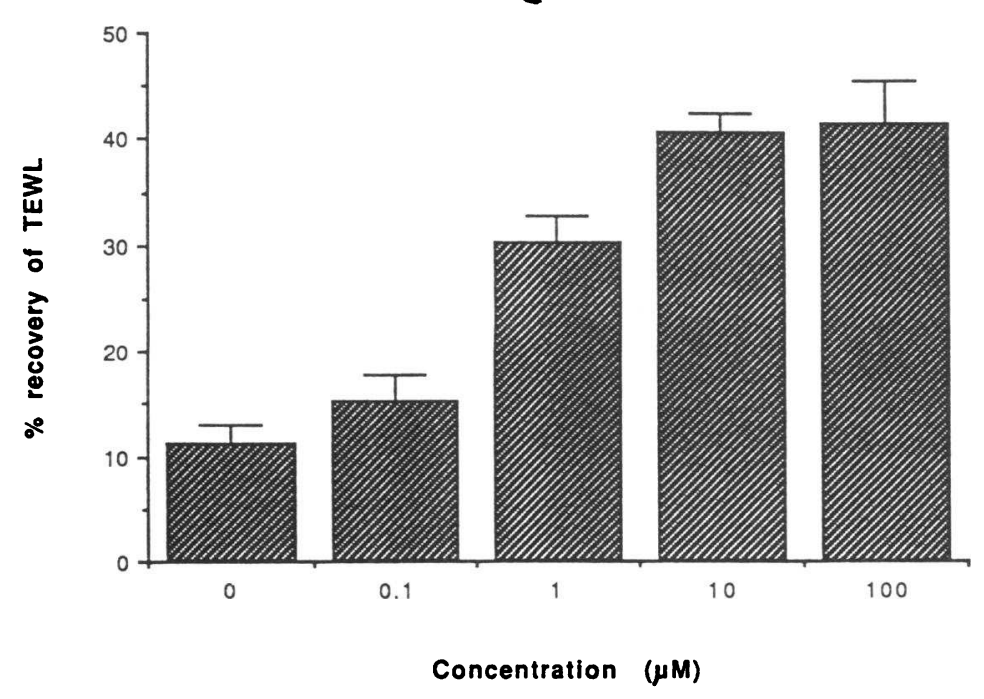

Figure 9. Effect of TMB-8 on barrier recovery. After acetone disruption of the barrier, mice were immersed in isoosmolar sucrose containing $1.8 \mathrm{mM} \mathrm{CaCl}_{2}$ and $40 \mathrm{mM}$ $\mathrm{KCl}$. Various concentrations of TMB- 8 were added as indicated and TEWL was determined at $2.5 \mathrm{~h}$. Data are presented and expressed as in earlier figures. $N=4-7$ for each group. 


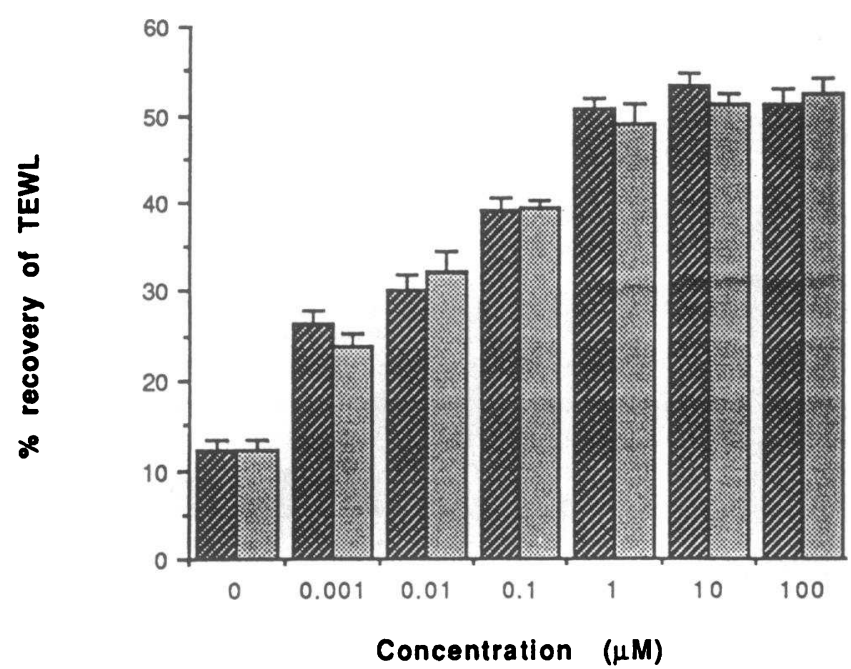

Figure 10. Effect of calmodulin antagonists on barrier recovery. After acetone disruption of the barrier, mice were immersed in isoosmolar sucrose containing $1.8 \mathrm{mM} \mathrm{CaCl}_{2}$ and $40 \mathrm{mM} \mathrm{KCl}$. Various concentrations of trifluoperazine or W7 were added as indicated and TEWL was determined at $2.5 \mathrm{~h}$. Data are presented and expressed as in earlier figures. $N=4-7$ for each group. $\square$, trifluoperazine; , W7.

with low calcium concentrations in the basal, proliferating layers, and progressively higher concentrations as one proceeds to the outer differentiated layers $(20,21)$. Whereas the low concentrations in the basal layers would be conducive to proliferation, the higher concentrations in the stratum spinosum and granular layers would favor differentiation. In very recent studies, we have demonstrated the depletion of calcium from the upper epidermis following acute disruption of the barrier (Menon, G. K., S. H. Lee, P. M. Elias, and K. R. Feingold, manuscript submitted for publication). Based on these observations, it is likely that a decrease in the calcium concentration in the upper epidermis is involved in initiating the repair process.

We suspect that the calcium gradient is disrupted after solvent treatment because of accelerated water transit leading to the increased passive loss of calcium. Disruption of the barrier is associated with increased ion flux across the skin (22). Moreover, artificial restoration of the barrier with a water vapor-impermeable membrane would prevent such water transit and loss of calcium. It should be recognized that while an impermeable membrane would inhibit the calcium loss by completely blocking water flux, immersion in isotonic or isoosmolar solutions, which prevents the net transport of water, would not inhibit either transcutaneous water fluxes or calcium loss. Thus, water loss, while not the direct signal for barrier repair, may be a necessary component to induce these changes in epidermal calcium concentration that in turn stimulate barrier repair.

Additional evidence of the importance of calcium is shown by the effect of inhibition of calcium transport on barrier recovery. Both verapamil and nifedipine, which are chemically unrelated and inhibit calcium transport into cells via the $L$ channel (9), prevent the calcium induced inhibition of barrier repair. This finding not only confirms a regulatory role for calcium in barrier repair, but also demonstrates that changes in intracellular calcium levels are important in mediating this inhibition of repair.
That cytosolic calcium is important is further shown by our experiments demonstrating that the inhibition of the transport of calcium from membrane storage pools to the cytosol by TMB-8 (10) prevents the inhibition of barrier recovery induced by calcium and potassium. Moreover, both trifluoperazine and W7, two structurally different calmodulin inhibitors $(11,12)$, also prevent the inhibition of barrier recovery. Taken together these results suggest a possible mechanism for the calcium effects; calcium enters the cell via $L$ channels, binds to calmodulin in the cytosol, and then by mechanisms yet to be elucidated inhibits lamellar body secretion and the repair response. Calcium calmodulin complexes have been shown to affect a large number of key cellular processes such as protein kinases, protein phosphatase-specific enzymes, ionic channels, etc. $(11,12)$.

Prior studies have shown that the lipids in the stratum corneum are primarily derived from the secretion of lamellar bodies by the cells of the stratum granulosum, and that lamellar body secretion is markedly increased following barrier disruption $(1,2,8)$. This rapid secretion of preformed lamellar bodies is followed by the synthesis of new lamellar bodies and further secretion (8). In most secretory systems, increases in intracellular calcium concentration stimulate secretion $(23,24)$. However, in keratinocytes it appears that increased intracellular calcium inhibits, while decreased intracellular calcium stimulates lamellar body secretion, analogous to the situation in the parathyroid gland where increased calcium concentrations decrease parathyroid hormone secretion while decreased calcium concentrations increase parathyroid hormone secretion (13). However, just as in the parathyroid gland, at this time the mechanism(s) by which the regulatory ions modulate lamellar body formation and secretion remain speculative.

Calcium alone, however, is only modestly effective in inhibiting barrier repair. Potassium alone and phosphate alone also produce a comparable modest inhibition of barrier repair. As occurs with calcium, it is likely that increased transepidermal water loss alters the epidermal potassium and phosphate concentration after barrier disruption due to increased passive loss. Studies by others have demonstrated increased potassium flux following disruption of the barrier (22). Calcium and potassium produce a synergistic inhibition of barrier recovery. These

Table I. HMG CoA Reductase Activity After Acetone Treatment

\begin{tabular}{lcc}
\hline \multicolumn{1}{c}{ Treatment } & Enzyme Activity & Percent change \\
\hline & $n$ mol/min per mg protein & $\%$ \\
Saline control $(n=4)$ & $0.629 \pm 0.020$ & - \\
Acetone - Air exposed $(n=3)$ & $1.038 \pm 0.072^{*}$ & +65 \\
Acetone - PBS $-\mathrm{Ca}^{++}$, & & \\
$\mathrm{Mg}^{++}$free $(n=7)$ & $0.905 \pm 0.029^{\ddagger}$ & +44 \\
Acetone $-\mathrm{PBS}+\mathrm{Ca}^{++}(n=4)$ & $0.607 \pm 0.065$ & -3 \\
\hline
\end{tabular}

After acetone disruption of the barrier, mice were left exposed to air or immersed in a calcium-, magnesium-free PBS or PBS + $1.8 \mathrm{mM}$ $\mathrm{CaCl}_{2}$ solution for $2.5 \mathrm{~h}$. A saline treated group of animals in which the barrier was not disturbed served as a control group. HMG CoA reductase activity in the epidermis was determined as described in Methods. The results are presented as mean \pm SEM. $N$, number of animals.

${ }^{*} P<0.01$ compared to control; ${ }^{\ddagger} P<0.001$ compared to control. 

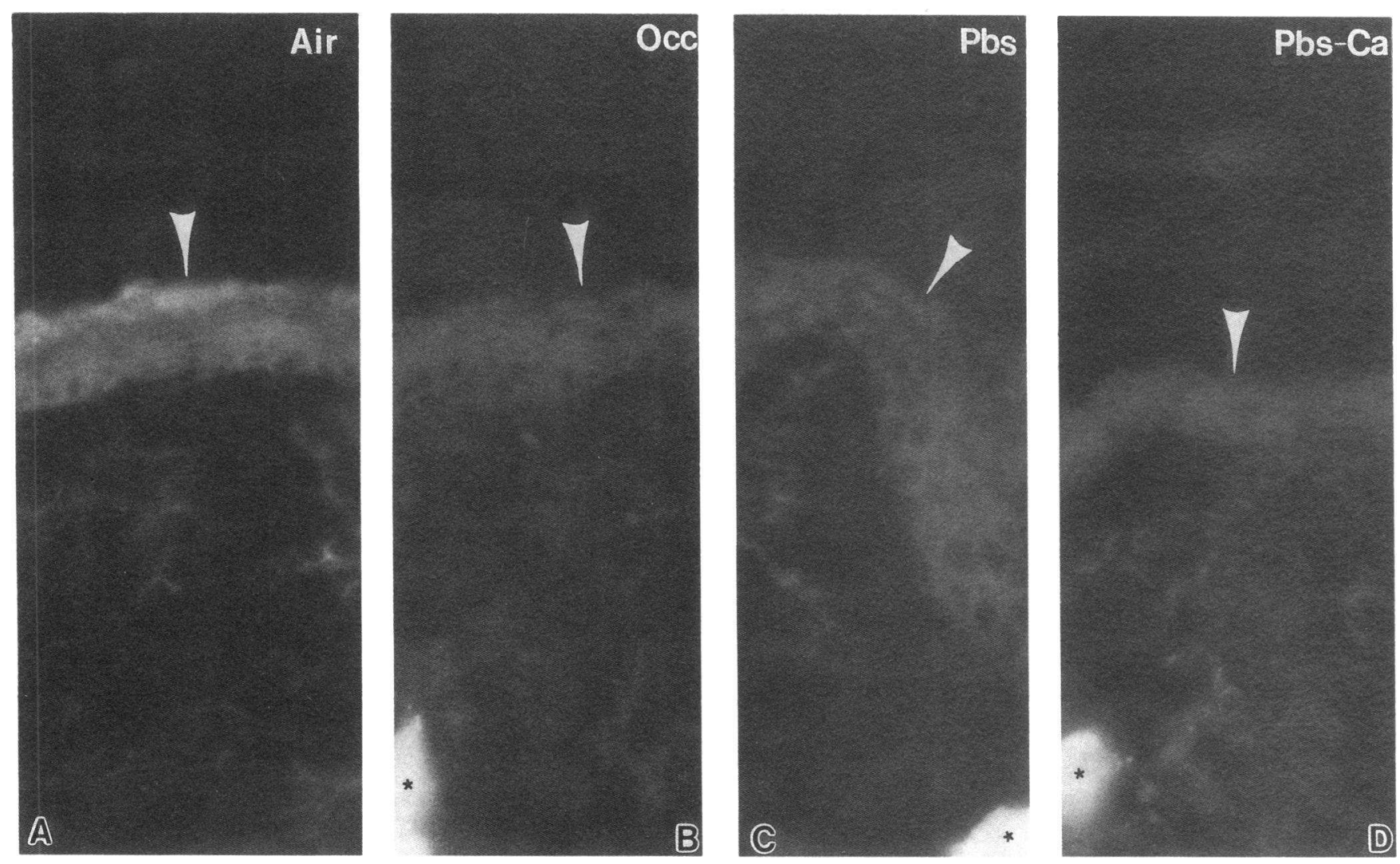

Figure 11. Lipid histochemistry after exposure to various solutions. $2.5 \mathrm{~h}$ after acetone treatment, followed by air exposure, there is a return of prominent green-gold fluorescence in the stratum corneum (arrows) with nile red staining $(A)$. When animals are occluded with a latex-wrap immediately after acetone treatment, no stainable lipid is evident $(B$, arrow). $2.5 \mathrm{~h}$ after immersion in PBS, partial return of green-gold fluorescence is apparent $(C)$. In contrast, in PBS plus calcium $(1.0 \mathrm{mM})$, little return of lipid staining is apparent $(D)$. In comparison note bright green-gold fluorescence of pilosebaccous lipids (asterisks) in dermis in $B-D$. Surface staining in $C$ is a result of secreted pilosebaceous lipids and does not contribute to the recovery of barrier function.

results are consistent with work in cultured keratinocytes, which have shown that intracellular potassium concentrations increase when calcium is added to the media $(25,26)$. Moreover, calcium-induced differentiation of keratinocytes in culture requires this increase in intracellular potassium $(25,26)$. If potassium levels in the media are decreased, the addition of calcium is unable to induce keratinocyte differentiation (25, 26). Similarly, the use of drugs, such as harmaline or TMB-8, that inhibit calcium-induced increases in intracellular potassium, also prevents calcium-induced differentiation $(25,26)$. The precise mechanism by which calcium leads to increased intracellular levels of potassium in keratinocytes is unknown, but it could be related to a variety of calcium-activated potassium channels (27). Further studies are required to define the complex interactions by which calcium, potassium, and phosphate regulate barrier repair.

In addition to blocking the normalization of barrier function, immersion in calcium and potassium solutions also inhibit the characteristic increase in HMG CoA reductase activity that occurs after barrier disruption, as well as the return of lipids to the stratum corneum. These results indicate that calcium and potassium ions may regulate a wide spectrum of metabolic events involved in barrier homeostasis. How these metabolic changes are linked and coordinated is unknown. It is clear, however, that the inhibition in barrier response mediated by calcium and potassium can not be attributed to nonspecific toxic effects. After removal from the calcium- and potassiumcontaining solutions, the barrier recovers rapidly and the rate of recovery is similar to that observed in animals exposed to air immediately following barrier disruption.

In summary, this study demonstrates that the repair of the epidermal barrier after solvent disruption can be prevented by immersion in solutions containing calcium and potassium. This suggests that the repair process induced by barrier disruption may be signalled by a decrease in the concentrations of these ions in the epidermis secondary to increased water flux and the passive loss of these ions.

\section{Acknowledgments}

We thank P. Herranz for editorial assistance and Drs. Dolores Shoback, Daniel Bikle, Cosmo Fraser, Carl Grunfeld, S. Pillai, S. Grayson, Walter Holleran, and Marvin Siperstein for helpful suggestions and advice.

This work was supported by National Institutes of Health grants AR 19098 and AR 39639, and the Medical Research Service, Veterans Administration. Dr. Proksch was a recipient of a grant from the Deutsche Forschungsgemeinschaft (DFG) Bonn-Bed Godesberg, West Germany, and the Paul G. Unna Foundation.

\section{References}

1. Elias, P. M. 1983. Epidermal lipids, barrier function and desquamation. $J$. Invest. Dermatol. 80:44-49. 
2. Elias, P. M., and K. R. Feingold. 1988. Lipid-related barriers and gradients in the epidermis. Ann. NY Acad. Sci. 548:4-13.

3. Menon, G. K., K. R. Feingold, A. H. Moser, B. E. Brown, and P. M. Elias 1985. De novo sterologenesis in the skin. II. Regulation by cutaneous barrier requirements. J. Lipid Res. 26:418-427.

4. Grubauer, G., P. M. Elias, and K. R. Feingold. 1989. Transepidermal water loss: the signal for recovery of barrier structure and function. J. Lipid Res. 30:323-334.

5. Grubauer, G., K. R. Feingold, and P. M. Elias. 1987. The relationship of epidermal lipogenesis to cutaneous barrier function. J. Lipid Res. 28:746-752.

6. Proksch, E., P. M. Elias, and K. R. Feingold. 1990. Regulation of 3-Hydroxy-3-methylglutaryl-coenzyme A reductase activity in murine epidermis: modulation of enzyme content and activation state by barrier requirements. $J$. Clin. Invest. 85:874-882.

7. Rodwell, V. W., K. C. Nordstrom, and J. H. Mitschelen. 1976. Regulation of HMG COA reductase. J. Lipid Res. 14:1-74.

8. Feingold, K. R., M. Mao-Quiang, G. K. Menon, S. Cho, B. E. Brown, and P. M. Elias. 1990. Cholesterol synthesis is required for cutaneous barrier function in mice. J. Clin. Invest. 86:696-702.

9. Triggle, D. J., and R. A. Janis. 1987. Calcium channel ligands. Annu. Rev. Pharmacol. Toxicol. 27:347-369.

10. Smith, R. J., and S. S. Iden. 1979. Phorbol myristate acetate induced release of granule enzyme from human neutrophils: inhibition by the calcium antagonist, 8(N,N-diethylamino)-octyl 3,4,5-trimethoxybenzoate hydrochloride. Biochem. Biophys. Res. Commun. 91:263-271.

11. England, P. J. 1986. Intracellular calcium receptor mechanisms. Br. Med. Bull. 42:375-383.

12. Villereal, M. L., and H. C. Palfrey. 1989. Intracellular calcium and cell function. Annu. Rev. Nutr. 9:347-376.

13. Brown, E. M. 1991. Extracellular $\mathrm{Ca}^{2+}$ sensing, regulation of parathyroid cell function and role of $\mathrm{Ca}^{2+}$ and other ions as extracellular (first) messengers. Physiol. Rev. 71:371-411.

14. Hennings, H., D. Michael, C. Cheng, P. Steinert, K. Holbrook, and S. H. Yuspa. 1980. Calcium regulation of growth and differentiation of mouse epidermal cells in culture. Cell. 19:245-254.

15. Hennings, H., P. Steinert, and M. M. Buxman. 1981. Calcium induction of transglutaminase and the formation of (gamma-glutamyl) lysine cross-links in cultured mouse epidermal cells. Biochem. Biophys. Res. Commun. 102:739-745.

16. Dykes, P. J., L. A. Jenner, and R. Marks. 1982. The effect of calcium on the initiation and growth of human epidermal cells. Arch. Dermatol. Res. 273:225-231.

17. Stanley, J. R., and S. H. Yuspa. 1983. Specific epidermal protein markers are modified during calcium-induced terminal differentiation. J. Cell Biol. 96:1809-1814

18. Boyce, S. T., and R. G. Ham. 1983. Calcium regulated differentiation of normal human epidermal keratinocytes in chemically defined clonal culture and serum-free serial culture. J. Invest. Dermatol. 81:33-40.

19. Yuspa, S. H., A. E. Kilkenny, P. M. Steinert, and D. R. Roop. 1989. Expression of murine epidermal differentiation markers is tightly regulated by restricted extracellular calcium concentrations in vitro. J. Cell Biol. 109:12071217.

20. Menon, G. K., S. Grayson, and P. M. Elias. 1985. Ionic calcium reservoirs in mammalian epidermis: ultrastructural localization by ion-capture cytochemistry. J. Invest. Dermatol. 84:508-512.

21. Forslind, B. Quantitative x-ray microanalysis of skin. 1987. Acta Dermato-Venereol. Suppl. 134:1-8.

22. Lo, J. S., H. A. Oriba, H. I. Maibach, and P. L. Bailin. 1990. Transepidermal potassium ion, chloride ion, and water flux across delipidized and cellophane tape-stripped skin. Dermatologica (Basel). 180:66-68.

23. Rubin, R.P. 1970. The role of calcium in the release of neurotransmitter substances and hormones. Pharmacol. Rev. 22:389-428.

24. Schoen, R. E., W. H. Frishman, and H. Shamoon. 1988. Hormonal and metabolic effects of calcium channel antagonists in man. Am. J. Med. 84:492502.

25. Hennings, H., K. A. Holbrook, and S. H. Yuspa. 1983. Factors influencing calcium-induced terminal differentiation in cultured mouse epidermal cells. J. Cell. Physiol. 116:265-281.

26. Hennings, H., K. A. Holbrook, and S. H. Yuspa. 1983. Potassium mediation of calcium-induced terminal differentiation of epidermal cells in culture. $J$. Invest. Dermatol. 81:50-55.

27. Latorre, R., A. Oberhauser, P. Labarca, and O. Alvarez. 1989. Varieties of calcium activated potassium channels. Annu. Rev. Physiol. 51:385-399. 